\title{
A Case Report Demonstrating the Potential Clinical Relevance of Liquid Tumor Biopsies in Lung Cancer
}

\author{
Revathi Suppiah $^{\mathrm{a}} \quad$ Bruce Gershenhorn $^{\mathrm{a}}$ Maurie Markman ${ }^{\mathrm{b}}$ \\ ${ }^{a}$ Cancer Treatment Centers of America, Midwestern Regional Medical Center, \\ Zion, IL, USA; ${ }^{b}$ Cancer Treatment Centers of America, Eastern Regional Medical Center, \\ Philadelphia, PA, USA
}

\section{Keywords}

Epidermal growth factor receptor · Lung cancer · Mutation

\begin{abstract}
A 50-year-old male with advanced non-small-cell lung cancer was unable to have standardof-care molecular testing performed at diagnosis as a result of inadequacy of the available tissue. A subsequently performed commercial liquid tumor biopsy (Foundation $A C T^{\circledR}$ ) revealed an epidermal growth factor receptor exon 19 deletion, but due to the progression of the tumor and rapid deterioration in the patient's performance status, a meaningful attempt at therapy directed to this recognized therapeutic target was not possible. This case provides important support for the relevance of liquid tumor biopsies in documenting highly clinically relevant molecular targets, particularly in the setting where limited solid tumor tissue is available for analysis.

\section{Introduction}

Standard-of-care management of advanced and metastatic non-small-cell lung cancer currently includes examination of the solid tumor for the presence of specific molecular abnormalities, which may have substantial impact on the choice of therapy $[1,2]$.

For patients presenting with advanced disease where surgical resection is not a therapeutic option, the overriding goal is to obtain a histologic diagnosis in as noninvasive a manner as possible and subsequently initiate therapy. However, the legitimate aim to avoid un- 


\section{Case Reports in Oncology}

necessary risk to a patient secondary to invasive procedures may conflict with the requirement to obtain sufficient viable cancer tissue to perform critical laboratory based analysis. It is in this setting where so-called 'liquid tumor biopsies' may be particularly relevant in disease management $[3,4]$.

The clinical course of a patient with advanced non-small-cell lung cancer recently seen in our cancer program demonstrates the critical importance of this rapidly developing paradigm.

\section{Case Presentation}

A 50-year-old African-American male never-smoker with no significant past medical history presented to his physician with a chief complaint of a dry cough during the summer of 2015 . He was treated with antibiotics, but the cough persisted.

In November 2015, a CT scan of the chest revealed a cavitary mass/infiltrate centered in the left lower lobe and left hilum. There was pathological lymphadenopathy in the mediastinum. The left pulmonary artery was narrowed by the left hilar mass with a positron emission tomography scan revealing intense activity in this region. Further evaluation revealed multifocal metastases, including bilateral supraclavicular lymph nodes, mediastinal lymph nodes, left lateral 8th rib, and left ischium osseous metastases. MRI of the brain demonstrated multiple small enhancing brain metastases.

A CT-guided lung biopsy revealed a poorly differentiated non-small-cell carcinoma with both adenocarcinoma and squamous cell carcinoma features. From the outside institution report, there was inadequate tissue sample for genomic testing.

The patient was treated with whole-brain radiation and a chemotherapy regimen consisting of cisplatin plus gemcitabine. Restaging scans in March 2016, after 3 cycles of cisplatin/gemcitabine, revealed progressive disease in the lungs. MRI of the brain demonstrated a decrease in all intracranial metastases.

In early March 2016, the patient was started on second-line treatment with the immunotherapeutic agent nivolumab, but subsequent evaluation revealed progression of the disease throughout the chest and new metastatic disease in the right adrenal gland. Positron emission tomography/CT documented multiple new bony metastatic lesions and innumerable metastatic nodules throughout the right lung. There was also a new hepatic lesion.

The patient presented to our institution in July 2016 for a second opinion. Due to significant respiratory distress, he was admitted to the hospital where his performance and respiratory status continued to decline significantly (fig. 1).

The patient had had a liquid biopsy (Foundation ACT ${ }^{\circledR}$ ) ordered by his prior oncologist shortly before he came to our institution, which revealed an epidermal growth factor receptor exon 19 deletion. When this was learned, he was started on afatinib, but unfortunately, his respiratory status deteriorated further the same day, requiring intubation. The patient expired several days later as a result of progressive respiratory failure.

\section{Discussion}

Lung cancer is the leading cause of cancer-related death. Despite advances in the discovery of genomic alterations, the overall 5-year survival rate is currently less than $20 \%$. 
Given the remarkable advances in targeted therapies for metastatic lung adenocarcinomas, tumor molecular profiling is essential at the time of diagnosis or at the time of relapse. Obtaining adequate tissue from the lung or metastatic sites can be limited due to patient's performance status or due to risks involved with the procedures.

Liquid tumor biopsies have stirred much excitement in the oncology world [3, 4]. Liquid biopsy involves a noninvasive approach to obtaining circulating tumor cells and nucleic acids including cell-free RNA, microRNA, and circulating cell-free DNA. Cancers that have 50 million malignant cells can release enough DNA for the detection of circulating tumor DNA in the blood. Levels of circulating tumor DNA can vary widely. Tumor size, type, stage of disease, sites of metastasis, and histologic grade may affect the levels of circulating DNA.

While blood-based testing can have a shorter turnaround time compared to tissuebased molecular analysis, existing data suggest that they have lower overall sensitivity. Currently, liquid biopsies should be considered only when tumor tissue is inadequate for molecular analysis and/or when the risk of a repeat biopsy is significant [4].

The case presented here demonstrates the potential major utility associated with liquid tumor biopsies in lung cancer. There was insufficient tissue available at diagnosis and as a result, the 'actionable mutation' present within our patient's cancer was not discovered until progressive cancer and rapid deterioration of his performance status prevented him from receiving a targeted approach that may have favorably influenced the clinical course.

The routine use of validated liquid tumor biopsies in the management of non-small-cell lung cancer should be considered in any case where the available 'solid' tissue does not permit essential evaluation for the presence of a clinically validated 'actionable' molecular target.

\section{Statement of Ethics}

The authors have no ethical conflicts to disclose.

\section{Disclosure Statement}

The authors have no conflicts of interest to disclose.

\section{References}

1 Zhou C, Wu Y-L, Chen G, et al: Erlotinib versus chemotherapy as first-line treatment for patients with advanced EGFR mutation-positive non-small-cell lung cancer (OPTIMAL, CTONG-0802): a multicenteropen-label, randomized phase 3 study. Lancet Oncol 2011;12:735-742.

-2 Solomon BJ, Mok T, Kim D-W, et al: First-line crizotinib versus chemotherapy in ALK-positive lung cancer. N Engl J Med 2014;371:2167-2177.

3 Alix-Panabieres C, Pantel K: Circulating tumor cells: liquid biopsy of cancer. Clin Chem 2013;59:110118.

4 Sholl LM, Aisner DL, Allen TC, et al: Liquid biopsy in lung cancer: a perspective from members of the Pulmonary Pathology Society. Arch Pathol Lab Med 2016;140:825-829. 


\section{Case Reports in Oncology}

Suppiah et al:: A Case Report Demonstrating the Potential Clinical Relevance of Liquid Tumor Biopsies in Lung Cancer

CT A/P 7-22-16

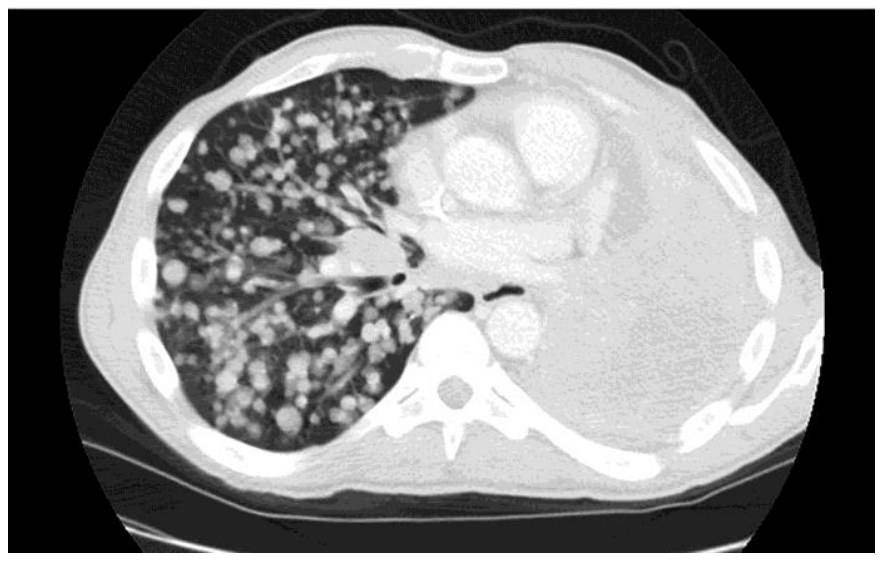

Fig. 1. Chest CT scan on admission. 